\title{
Functional characterization of the gene promoter for an Elaeis guineensis phosphate starvation-inducible, high affinity phosphate transporter in both homologous and heterologous model systems
}

\begin{abstract}
Oil palm is grown in tropical soils with low bioavailability of Pi. A cDNA clone specifically expressed under phosphate-starvation condition in oil palm roots was identified as a high-affinity phosphate transporter (EgPHT1). The deduced amino acid sequence has 6 transmembrane domains each at the $\mathrm{N}$ - and C-termini separated by a hydrophilic linker. Comparison of promoter motifs within $1500 \mathrm{bp}$ upstream of ATG of 10 promoters from high- and low-affinity phosphate transporter from both dicots and monocots including EgPHT1 was performed. The EgPHT1 promoter was fused to $\beta$ glucuronidase (GUS) reporter gene and its activity was analysed by histochemical and fluorometric GUS assays in transiently transformed oil palm tissues and T3 homozygous transgenic Arabidopsis plants. In response to Pi-starvation, no GUS activity was detected in oil palm leaves, but a strong inducible activity was observed in the roots (1.4 times higher than the CaMV35S promoter). GUS was specifically expressed in transgenic Arabidopsis roots under Pi deficiency and starvation of the other macronutrients ( $\mathrm{N}$ and $\mathrm{K}$ ) did not induce GUS activity. Eight motifs including ABRERATCAL (abscisic-acid responsive), RHERPATEXPA7 (root hair-specific), SURECOREATSULTR11 (sulfur-deficiency response), LTRECOREATCOR15 (temperature-stress response), MYB2CONSENSUSAT and ACGTATERD1 (waterstress response) as well as two novel motifs, 3 (TAAAAAAA) and 26 (TTTTATGT) identified through pattern discovery, occur at significantly higher frequency $(p<0.05)$ in the high-than the low-affinity phosphate transporter promoters. The Pi deficiencyresponsive elements in EgPHT1 includes the P1BS, W-box, E-box and the G-box. Thus, EgPHT1 is important for improving Pi uptake in oil palm with potential for engineering efficient $\mathrm{Pi}$ acquisition.
\end{abstract}

Keyword: High-affinity phosphate transporter; Oil palm; Phosphate starvation; Promoter analysis; Transgenic Arabidopsis 
